\title{
Micron-Scale NV-NMR Spectroscopy with Signal Amplification by Reversible Exchange
}

\author{
Nithya Arunkumar, ${ }^{1,2,3}$ Dominik B. Bucher, ${ }^{1,3,4}$ Matthew J. Turner, ${ }^{1,5}$ Patrick TomHon, ${ }^{6}$ David Glenn, ${ }^{1}$ \\ Sören Lehmkuhl, ${ }^{6}$ Mikhail D. Lukin, ${ }^{1}$ Hongkun Park® ${ }^{1,7}$ Matthew S. Rosen $\circledast,{ }^{1,8,9}$ \\ Thomas Theis $\odot,{ }^{6,10,11}$ and Ronald L. Walsworth ${ }^{1,2,3,5,12,13, *}$ \\ ${ }^{1}$ Department of Physics, Harvard University, Cambridge, Massachusetts 02138, USA \\ ${ }^{2}$ Quantum Technology Center, University of Maryland, College Park, Maryland 20742, USA \\ ${ }^{3}$ Harvard-Smithsonian Center for Astrophysics, Cambridge, Massachusetts 02138, USA \\ ${ }^{4}$ Department of Chemistry, Technical University of Munich, Munich, Germany \\ ${ }^{5}$ Center for Brain Science, Harvard University, Cambridge, Massachusetts 02138, USA \\ ${ }^{6}$ Department of Chemistry, North Carolina State University, Raleigh, North Carolina 27695, USA \\ ${ }^{7}$ Department of Chemistry and Chemical Biology, Harvard University, Cambridge, Massachusetts 02138, USA \\ ${ }^{8}$ A. A. Martinos Center for Biomedical Imaging, Massachusetts General Hospital, Boston, Massachusetts 02129, \\ USA \\ ${ }^{9}$ Harvard Medical School, Boston, Massachusetts 02129, USA \\ ${ }^{10}$ Department of Physics, North Carolina State University, Raleigh, North Carolina 27695, USA \\ ${ }^{11}$ UNC \& NC State Joint Department of Biomedical Engineering, Raleigh, North Carolina 27695, USA \\ ${ }^{12}$ Department of Physics, University of Maryland, College Park, Maryland 20742, USA \\ ${ }^{13}$ Department of Electrical and Computer Engineering, University of Maryland, College Park, Maryland 20742, \\ USA
}

(Received 21 June 2020; accepted 11 November 2020; published 11 January 2021)

\begin{abstract}
Optically probed nitrogen-vacancy (NV)) quantum defects in diamond can detect nuclear magnetic resonance (NMR) signals with high-spectral resolution from micron-scale sample volumes of about $10 \mathrm{pl}$. However, a key challenge for NV-NMR spectroscopy is detecting samples at millimolar concentrations. Here we demonstrate an increase in NV-NMR proton concentration sensitivity by hyperpolarizing sample proton spins to about $0.5 \%$ through signal amplification by reversible exchange (SABRE), enabling micron-scale NMR spectroscopy of small-molecule sample concentrations as low as $1 \mathrm{mM}$ in picoliter volumes. The SABRE-enhanced NV-NMR technique may enable detection and chemical analysis of low-concentration molecules and their dynamics in complex micron-scale systems such as single cells.
\end{abstract}

DOI: 10.1103/PRXQuantum.2.010305

Nitrogen-vacancy (NV) quantum defects in diamond are a leading modality for sensitive magnetometry with high spatial-resolution and operation under ambient conditions [1,2], including for nuclear magnetic resonance (NMR) spectroscopy at small length scales (nanometers to microns) [3-8]. Initial work on NV-NMR spectroscopy [3-8] suffered from low spectral resolution (kilohertz) due to the short decoherence time of the NV centers. To

\footnotetext{
*walsworth@umd.edu
}

Published by the American Physical Society under the terms of the Creative Commons Attribution 4.0 International license. Further distribution of this work must maintain attribution to the author(s) and the published article's title, journal citation, and DOI. overcome this problem, Glenn et al. [9] implemented a coherently averaged synchronized readout (CASR) technique and demonstrated a NV-NMR spectral resolution of a few hertz at $88 \mathrm{mT}$ for a micron-scale sensing volume. However, because of the finite sensitivity of the NV-NMR sensor, its application is restricted to highly concentrated pure samples, which limits its utility for most chemical and biological problems.

Recently, Bucher et al. [10] used dynamic nuclear polarization (DNP) based on the Overhauser mechanism [11], where polarization is transferred to the sample nuclear spins from the electronic spins of dissolved molecular radicals, and obtained a proton number sensitivity enhancement of 2 orders of magnitude for micron-scale CASR NV-NMR spectroscopy. However, such DNP sensitivity enhancement is limited by the finite electronic spin 
polarization at the moderate magnetic fields $(3 \mathrm{~T}$ and below [8]) and ambient temperatures used for NV-NMR spectroscopy. Higher sample nuclear spin polarization and thereby an increase in NMR sensitivity can be achieved through a parahydrogen-based signal amplification by reversible exchange (SABRE) process [12-14]. To date, SABRE has been shown to enhance conventional inductive NMR sensitivity in microliter-scale to milliliter-scale sensing volumes [12-17].

Here we integrate SABRE hyperpolarization with CASR NV-NMR spectroscopy to realize about $0.5 \%$ proton spin polarization in a micron-scale sample at $6.6 \mathrm{mT}$, providing an enhancement of concentration sensitivity of 5 orders of magnitude relative to thermal spin polarization at this bias field. Using our method, we measure the NMR spectrum of small-molecule samples with concentrations as low as $1 \mathrm{mM}$ and for a sensing volume of $10 \mathrm{pl}$. The high spectral resolution of SABRE-enhanced NV-NMR spectroscopy also enables the measurement of $J$ couplings in dilute molecules, thereby providing chemical specificity.

The experimental setup is shown in Fig. 1(a). The NVNMR sensor is a $2 \times 2 \times 0.5 \mathrm{~mm}^{3}$ high-purity diamond chip with a $13-\mu \mathrm{m}$-thick NV layer and a NV concentration of $3 \times 10^{17} \mathrm{~cm}^{-3}$. The [111] axis of the NV-NMR sensor is oriented parallel to the bias magnetic field $\left(B_{0} \approx 6.6\right.$ $\mathrm{mT})$, which is generated by a feedback-stabilized electromagnet [9]. A green optical beam $(\lambda=532 \mathrm{~nm})$ in a total internal reflection configuration with a spot diameter of approximately $15 \mu \mathrm{m}$ is used to initialize and read out the electronic spins of the NV-NMR sensor [9]. A single-coil wire loop antenna [18], placed directly above the NV surface of the diamond, is used to drive the electron spin resonance transitions of the NV centers. At the proton NMR frequency of $280 \mathrm{kHz}$ for a $6.576-\mathrm{mT}$ bias field, the ac magnetic field sensitivity $\left(\eta_{B}\right)$ of the NV ensemble sensor is $35(2) \mathrm{pT} / \sqrt{\mathrm{Hz}}$. The liquid sample is placed directly on top of the diamond surface. The thickness of the NV layer and the spot diameter of the optical beam provide an effective NMR sensing volume of about $10 \mathrm{pl}[9,10]$.

Hyperpolarization of proton spins in the sample molecules is obtained through SABRE. Parahydrogen gas is first dispersed into the sample solution [Fig. 1(a)] for about $20 \mathrm{~min}$ to activate an iridium-based catalyst, which then mediates reversible exchange of spin order between the parahydrogen and the small-molecule substrate - the sample to be probed with NV-NMR

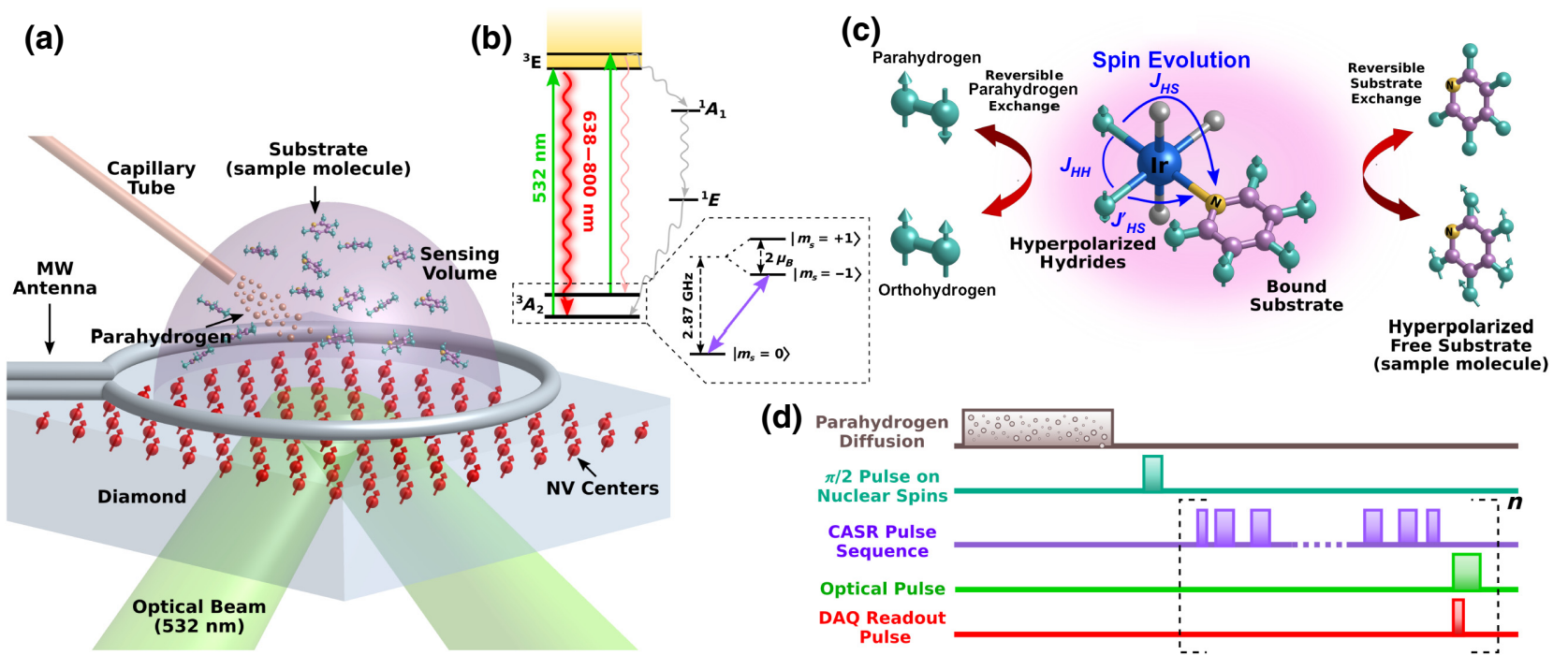

FIG. 1. NV-NMR sensor integrated with SABRE. (a) Experimental schematic of the ensemble NV-NMR sensor. A 532-nm optical beam illuminates the diamond chip via a total internal reflection configuration. A microwave (MW) antenna on the diamond chip drives the electronic spins of the NV centers. Parahydrogen diffused into the sample through the capillary tube initiates the SABRE reaction, and thereby hyperpolarizes sample proton spins via an iridium-based catalyst in the sample solution. The hyperpolarized NMR signal is sensed with use of the electronic spins of the ensemble of NV centers. (b) Energy level diagram for the NV centers in diamond. The expanded view shows the Zeeman splitting of the ground triplet state ${ }^{3} A_{2}$ in the presence of a magnetic field. (c) SABRE hyperpolarization process. The SABRE catalyst is in reversible exchange (indicated by red arrows) with parahydrogen (left side) and a small-molecule substrate (e.g., pyridine; right side). In the transient (millisecond) bound state of the catalyst-substrate complex (center), spin order flows from the hydrides to the substrate, leading to the buildup of polarization on the free substrate in solution, which is the sample to be probed with NV-NMR spectroscopy. (d) Pulse sequence for SABRE hyperpolarization and NV-NMR detection. The parahydrogen, bubbled into the sample solution, activates the catalyst and hyperpolarizes the small-molecule substrate (the sample). A $\pi / 2$ pulse induces a FNP signal from the hyperpolarized sample, which is detected by NV sensor spins via a CASR pulse sequence. DAQ, data acquisition. 
spectroscopy - as shown in Fig. 1(c). Once activated, about $30 \mathrm{~s}$ of additional parahydrogen bubbling is sufficient to establish hyperpolarization on the substrate. During the transient lifetime of the catalyst-substrate complex (on the order of milliseconds), proton spin order flows from the hydrides (in parahydrogen) to protons in the bound small-molecule substrate (e.g., pyridine). Lastly, the hyperpolarized substrate dissociates to give free hyperpolarized small molecules in solution with polarization lifetime $T_{1} \sim 5 \mathrm{~s}$. The polarization transfer process is resonant at about $6.6 \mathrm{mT}$, where the $J$ coupling between the hydrides equals the frequency difference between hydride and substrate proton spins, leading to a level anticrossing between the singlet state of the hydrides and the proton spin-down states of the substrate. In summary, spin evolution and chemical exchange continually pump hyperpolarization into free small molecules in solution as long as the parahydrogen is periodically refreshed by bubbling between NV-NMR measurements. Details of the homebuilt parahydrogen generation, its integration with the NV-NMR sensor, and the SABRE polarization transfer mechanism are discussed in the Appendix. Also, the efficiency of the SABRE technique as a function of the bias magnetic field was characterized previously [13].

After a waiting time of $1 \mathrm{~s}$ following SABRE hyperpolarization, a $\pi / 2 \mathrm{rf}$ pulse is applied resonant with the nuclear spins of the sample. The induced Larmor precession of the nuclear spin results in a decaying oscillatory magnetic field called "free nuclear precession" (FNP). The NV-NMR sensor is then probed with a CASR pulse sequence [9], which detects the FNP signal and maps it onto a population difference of the NV ensemble electron spin states. The population difference is read out optically by spin state-dependent fluorescence for $1 \mu \mathrm{s}$, followed by optical reinitialization of the NV electronic spins for $4 \mu \mathrm{s}$.

As a first demonstration we detect the SABRE-enhanced NV-NMR spectrum of a sample of pyridine, a weakly alkaline heterocyclic organic molecule. The sample solution is made with $100 \mathrm{mM}$ pyridine and $5 \mathrm{mM}$ catalyst dissolved in methanol. We also apply a calibrated test ac magnetic signal using a coil antenna. The observed NVNMR spectrum with (red dots) and without (blue dots) SABRE hyperpolarization is shown in Fig. 2. The expected thermally polarized NV-NMR signal amplitude (without SABRE hyperpolarization) is $32 \mathrm{fT}$ at a bias magnetic field of $6.6 \mathrm{mT}$. The measured SABRE-enhanced NVNMR signal has a FWHM linewidth of 2.3(5) Hz and an amplitude of 7.1(1) nT (by comparison with the amplitude of the test signal), which is an enhancement of about $2.22(3) \times 10^{5}$ in signal amplitude over the expected thermally polarized signal, implying a proton spin polarization of $0.503(7) \%$ with the SABRE technique. The SNR of this single-shot hyperpolarized NMR signal is $320(3)$ for a measurement duration of $2 \mathrm{~s}$. This result corresponds to a molecule number sensitivity of $13.3(3) \mathrm{fmol} / \sqrt{\mathrm{Hz}}$

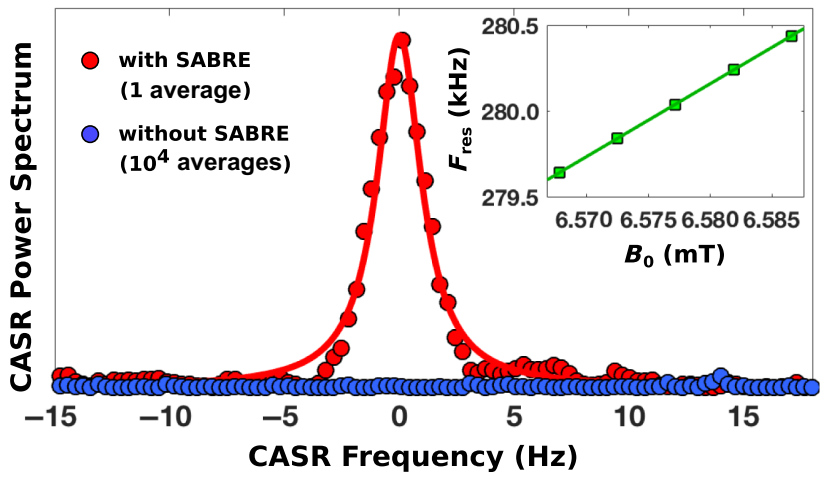

FIG. 2. SABRE-enhanced NV-NMR spectra of pyridine. Comparison of measured NV-NMR spectra of $100 \mathrm{mM}$ pyridine sample with (red circles, one acquisition) and without (blue circles, $10^{4}$ acquisitions) SABRE hyperpolarization for a CASR pulse sequence duration of $2 \mathrm{~s}$. The solid red line is a Lorentzian fit to the SABRE-enhanced NV-NMR spectrum, giving a linewidth of $2.3(5) \mathrm{Hz}$, a signal enhancement of about $2.22(3) \times 10^{5}$, and a proton spin polarization of $0.503(7) \%$, with a proton number sensitivity of $66.48(15) \mathrm{fmol} / \sqrt{\mathrm{Hz}}$ for a SNR of 3 . Small side peaks in the CASR spectra and deviations from the Lorentzian fits may arise from technical artifacts in the experiment [10]. The inset shows the CASR resonance frequency $F_{\text {res }}$ of hyperpolarized pyridine (green squares) obtained by our varying the bias magnetic field $B_{0}$. A linear fit (green line) of $F_{\text {res }}$ versus $B_{0}$ gives $\gamma_{p}=42.5355(40) \mathrm{MHz} / \mathrm{T}$, consistent with the proton gyromagnetic ratio.

for pyridine and a proton number sensitivity of $66.48(15)$ $\mathrm{fmol} / \sqrt{\mathrm{Hz}}$, which is an increase in proton number sensitivity of 2 orders of magnitude compared with the Overhauser DNP technique applied to NV-NMR spectroscopy [10]. The sensitivity is defined relative to a SNR of 3, which is typical in conventional NMR spectroscopy [19]. The pressure, flow rate, and parahydrogen bubbling duration are optimized to achieve this enhancement in sensitivity. The NV-NMR signal without hyperpolarization (blue dots in Fig. 2) is too weak to observe even after $10^{4}$ averages. We verify the hyperpolarized pyridine NV-NMR signal by measuring the signal resonance frequency $F_{\text {res }}$ as a function of the applied bias magnetic field $B_{0}$, yielding a variation of $\gamma_{p}=42.5355(40) \mathrm{MHz} / \mathrm{T}$, consistent with the gyromagnetic ratio of the proton (see Fig. 2, inset).

We next perform SABRE-enhanced NV-NMR spectroscopy (Fig. 3) by further diluting pyridine in methanol. Samples are prepared at pyridine concentrations of 100 , $30,10,5$, and $1 \mathrm{mM}$ in methanol. The concentration ratio of pyridine to the catalyst (20:1) is kept constant [20]. A hyperpolarized NV-NMR spectrum is observed even at a concentration of $1 \mathrm{mM}$ (10 fmol of pyridine molecules) with a SNR of 50 after averaging for $300 \mathrm{~s}$ [Fig. 3(a)]. The red dots in Fig. 3(b) denote the detected NV-NMR signal amplitude at various pyridine concentrations. The 
(a)

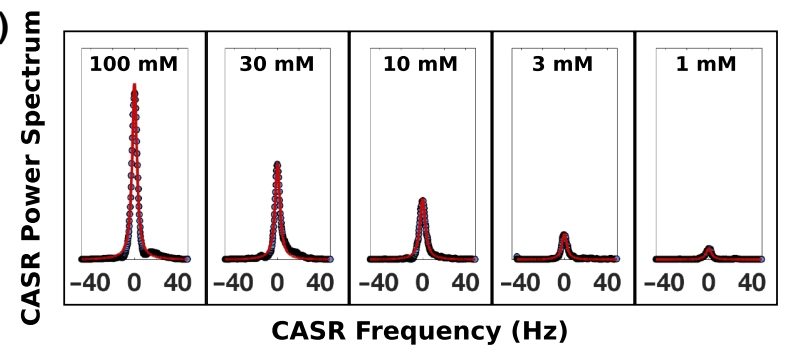

(b)

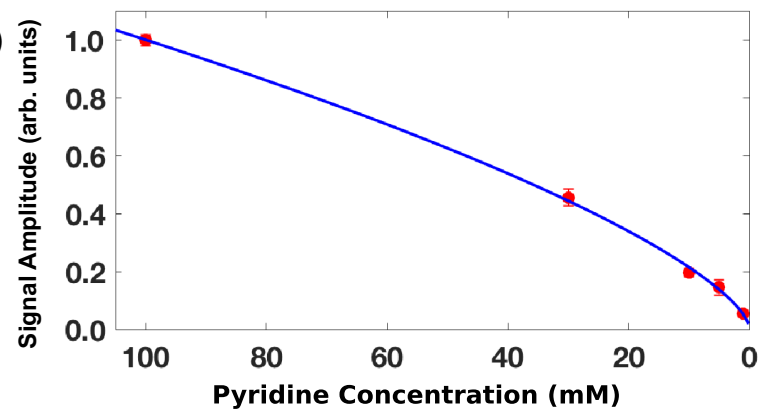

FIG. 3. SABRE-enhanced NV-NMR measurement for various pyridine concentrations. (a) CASR-detected NV-NMR spectra (blue circles) for hyperpolarized pyridine samples and the associated Lorentzian fits (solid red line) at concentrations of 100, $30,10,5$, and $1 \mathrm{mM}$ in methanol. (b) CASR-detected NV-NMR signal amplitude (red dots) of hyperpolarized pyridine at various concentrations. The solid blue curve is a power function model of the form $a x^{b}+c$ with fit parameters $a=0.042, b=0.6855$, and $c=0.012$.

error bars represent the standard deviation of the NVNMR signal measured across three independent trials. A power function model of the form $a x^{b}+c$ is fit to the experimental data [Fig. 3(b), solid blue curve], in excellent agreement with the measurements for fit parameters $a=0.042, b=0.6855$, and $c=0.012$. Deviations from a linear dependence are expected since SABRE hyperpolarization of fewer substrate molecules is more efficient than hyperpolarization of more substrate molecules [13,20,21], and thus the relative hyperpolarization decreases with increasing pyridine concentration. Developing a predictive physical model of this behavior is beyond the scope of the present investigation and is left to future work. Nonetheless, the model fit in Fig. 3(b) is repeatable and could be used, for a given SABRE NV-NMR system and within a calibrated concentration range, to quantify samples of unknown pyridine concentration.

To illustrate the versatility of our technique, we acquire SABRE-enhanced NV-NMR signals from two additional molecules (Fig. 4). First, we study ${ }^{15} \mathrm{~N}$-labeled pyridine, which has a $J$ coupling of about $10 \mathrm{~Hz}$ between the nuclear spins of the protons and the ${ }^{15} \mathrm{~N}$ atom $[17,21]$. The sample is prepared with $100 \mathrm{mM}{ }^{15} \mathrm{~N}$-labeled pyridine and 5 $\mathrm{mM}$ catalyst dissolved in methanol. The hyperpolarized NV-NMR spectrum has a SNR of 150(5) [Fig. 4(a)] for a

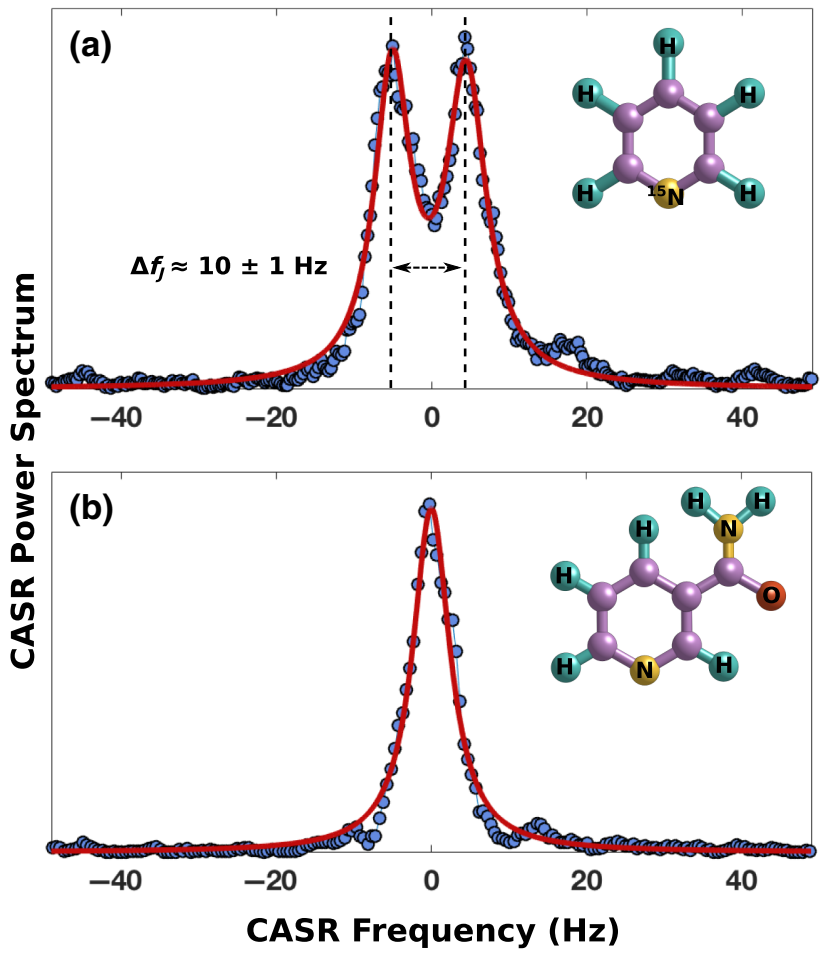

FIG. 4. SABRE-enhanced molecular NV-NMR spectra. (a) Single-shot CASR spectrum of hyperpolarized ${ }^{15} \mathrm{~N}$-labeled pyridine (blue circles) for a sensing duration of $3 \mathrm{~s}$ at a concentration of $100 \mathrm{mM}$. The double Lorentzian fit (solid red line) indicates a splitting of $\Delta f_{J} \approx 10(1) \mathrm{Hz}$ (indicated by the vertical dashed lines) due to $J$ coupling between the ${ }^{15} \mathrm{~N}$ nucleus and the protons. The inset shows the chemical structure of ${ }^{15} \mathrm{~N}$-labeled pyridine. (b) Single-shot CASR spectrum of hyperpolarized nicotinamide (blue circles) for a sensing duration of $2 \mathrm{~s}$ at a concentration of $100 \mathrm{mM}$. The Lorentzian fit (solid red line) gives a spectral linewidth of approximately 4(1) Hz. The inset shows the chemical structure of nicotinamide. Small side peaks in the CASR spectra and deviations from the Lorentzian fits may arise from technical artifacts in the experiment [10].

CASR pulse sequence duration of $3 \mathrm{~s}$. This high-resolution spectrum has a linewidth of $3(1) \mathrm{Hz}$ and shows wellresolved peaks due to the $J$ coupling [22], with a splitting of $\Delta f_{J} \approx 10(1) \mathrm{Hz}$ [Fig. 4(a), solid red line] determined from a double Lorentzian fit. Finally, we measure the SABRE-enhanced NV-NMR spectrum of nicotinamide, a water-soluble form of vitamin $\mathrm{B}_{3}$ (niacin), at $100 \mathrm{mM}$ concentration. The observed NMR spectrum has a SNR of 200(4) [Fig. 4(b)] for a CASR pulse sequence duration of 2 s. The spectral linewidth from the Lorentzian fit [Fig. 4(b), solid red line] is 4(1) Hz.

Nicotinamide has important functions in mammalian metabolism and is a metabolic precursor to oxidized nicotinamide adenine dinucleotide $\left(\mathrm{NAD}^{+}\right)$/reduced nicotinamide adenine dinucleotide (NADH) [23-25]. With further development, we envision using our technique to observe the conversion of nicotinamide to $\mathrm{NAD}^{+} / \mathrm{NADH}$, 
which could allow NMR measurement of the redox status in cells. Hyperpolarized NV-NMR spectroscopy may also enable metabolic studies of healthy and diseased cells with dysregulated metabolism on the single-cell level. To increase the chemical specificity necessary for such applications, several methods have recently been developed [26-33] that allow implementation of SABRE hyperpolarization at a tesla-scale bias magnetic field without the need for technically demanding field cycling. For example, rfSABRE methods [31-33] apply NMR pulse sequences to the catalyst-substrate complex spins, allowing polarization transfer from parahydrogen-derived hydrides to substrate molecules at any magnetic field. In future work, the hyperpolarization realized in the current NV-NMR experiment (about $0.5 \%$ ) could be further increased by implementation of the SABRE method on a microfluidic diamond chip, thereby increasing the contact area between the substrate and the parahydrogen $[34,35]$. An additional improvement in hyperpolarization of a factor of 3 can also be obtained by using pure parahydrogen instead of the 50\% parahydrogen produced by our homebuilt system.

In summary, we integrate micron-scale NV-NMR spectroscopy with the hyperpolarization of sample proton spins to about $0.5 \%$ through the SABRE technique. This advance augments the growing toolbox of techniques for sensitive, high-resolution NMR spectroscopy in micronscale samples using NV quantum defects in diamond. Compared with other signal enhancement methods, such as room temperature Overhauser DNP [10] or direct flow-based prepolarization [35], SABRE provides significantly higher concentration sensitivity $(1 \mathrm{mM}$ in the present example) while being applicable to a wide range of small-molecule analytes [20,36,37]. With planned extension to tesla-scale magnetic fields, SABRE-enhanced NVNMR spectroscopy may become a high-impact tool for biological applications, such as tracking and monitoring of chemical reactions of metabolites in single cells.

\section{ACKNOWLEDGMENTS}

We thank Edward Soucy and Brett Graham for technical support and fabrication assistance. This project is supported by, or in part by, the U.S. Army Research Laboratory and the U.S. Army Research Office under Grant Nos. W911NF1510548 and W911NF1920181, as well as the Moore Foundation. D.B.B. was partially supported by the German Research Foundation (BU 3257/ 1-1). The authors declare no competing interests.

M.S.R. and T.T. designed the SABRE technique and the homebuilt parahydrogen generator. S.L. synthesized the catalyst. S.L. and P.T. designed the method for SABRE sample preparation under inert gas conditions. N.A., D.B.B., M.J.T., and D.R.G. modified the NV-NMR spectrometer for the SABRE technique. N.A. and D.B.B. performed the experiments and analyzed the data. M.D.L,
H.P., and R.L.W. conceived the application of NV diamond magnetometry to NMR detection at short length scales. M.S.R., T.T., and R.L.W. supervised the project, including development of the overall experimental design. All authors discussed the results and participated in writing the manuscript.

\section{APPENDIX A: PARAHYDROGEN GENERATION}

We generate parahydrogen using a homebuilt system, as illustrated in Fig. 5. Room temperature hydrogen gas, consisting of $25 \%$ parahydrogen and $75 \%$ orthohydrogen, fills a copper tube filled with an iron oxide hydroxide $[\mathrm{FeO}(\mathrm{OH})]$ catalyst bed. The copper tube is then submerged in liquid nitrogen at approximately $77 \mathrm{~K}$. The iron oxide hydroxide catalyst induces rapid thermalization by allowing interconversion of orthohydrogen to parahydrogen on the catalyst surface. Following enrichment at low temperature for $1 \mathrm{~min}$, the gas can be handled at room temperature for several hours (up to days) without loss of the enriched parahydrogen fraction. The parahydrogen gas is then bubbled at a rate of approximately $75 \mathrm{sccm}$ through the SABRE solution in an optical cuvette, and the smallmolecule substrate is thereby hyperpolarized (details are provided in Appendix B). The parahydrogen gas is maintained at a room temperature pressure of $30 \mathrm{psi}$ before the flow controller for all the experiments described in the main text. To maintain the parahydrogen pressure and to avoid any parahydrogen leak, we ensure a gastight seal throughout the setup.

\section{APPENDIX B: SABRE HYPERPOLARIZATION PROCESS}

\section{Chemical activation}

The iridium-based catalyst used in SABRE hyperpolarization is $\{[\mathrm{IrCl}(\mathrm{COD})(\mathrm{IMes})](\mathrm{COD}=1,5$-cyclooctadiene; IMes = 1,3-bis(2,4,6-trimethylphenyl)imidazol2-ylidene)\}. As illustrated in Fig. 6, the SABRE catalyst precursor is chemically activated by our supplying hydrogen gas and a substrate (e.g., pyridine or nicotinamide). Hydrogen undergoes oxidative addition onto the iridium, and the COD in the catalyst precursor is hydrogenated to cyclooctane. Hence the COD will no longer interact with the catalyst. Instead, the substrate coordinates with the iridium to form the active spin-order transfer catalyst. Although enriched parahydrogen gas is used to activate the catalyst for experimental convenience, the spin state of the hydrogen gas is irrelevant during the activation process.

\section{Reversible exchange}

After chemical activation of the catalyst (Appendix B 1), parahydrogen and the small-molecule substrate undergo reversible exchange with the catalyst (Fig. 7). In the sample solution there are free (unbound) hydrogen, free 


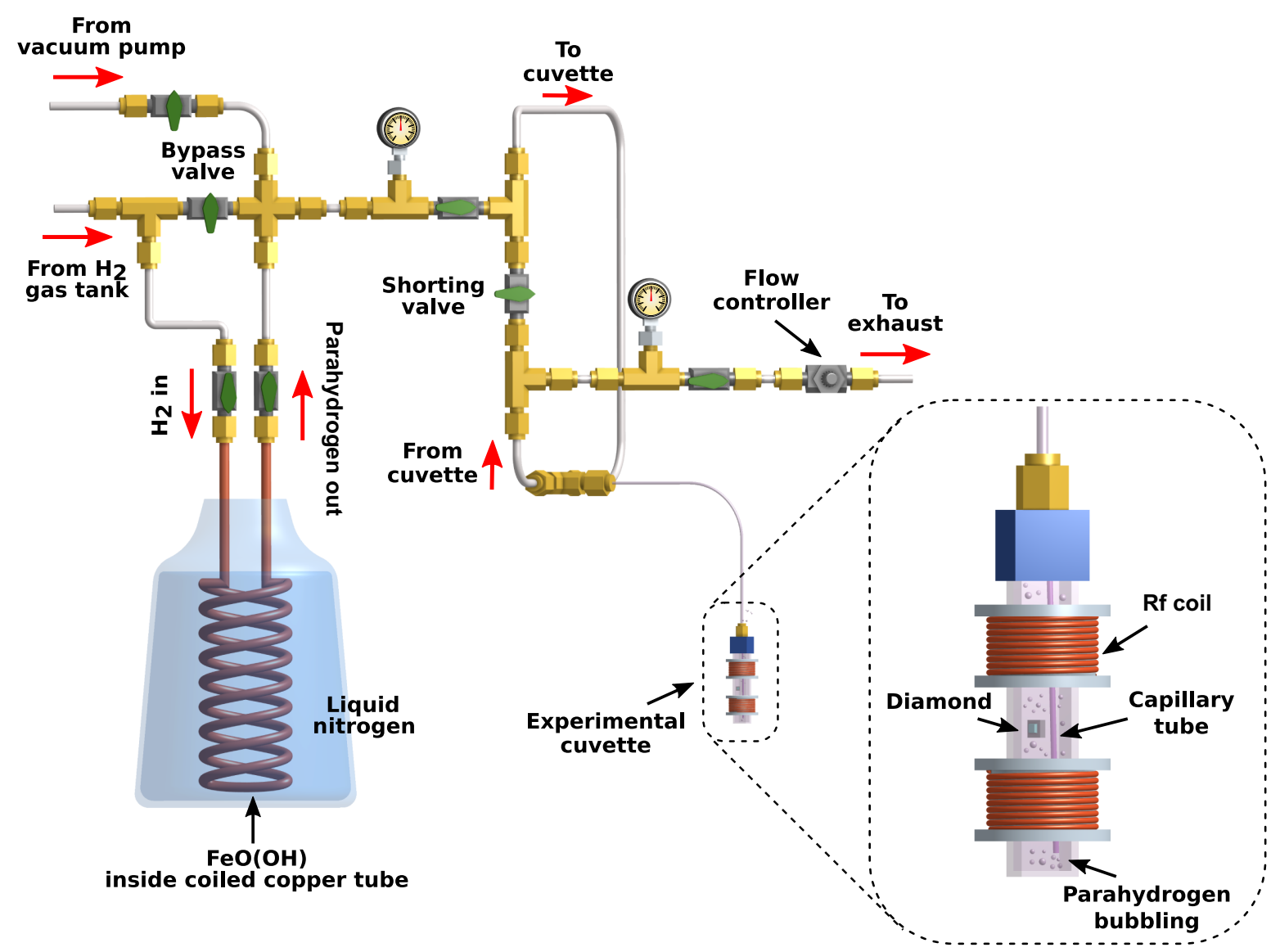

FIG. 5. Experimental apparatus for parahydrogen production and delivery to the NV-NMR setup ("experimental cuvette"). Parahydrogen is produced by our flowing hydrogen gas through an iron oxide catalyst at $77 \mathrm{~K}$. The parahydrogen is then bubbled through the capillary tube located within the experimental cuvette, where it interacts with the SABRE catalyst and hyperpolarization of the small-molecule substrate occurs. Optical NV-NMR detection is performed with a diamond chip integrated with the capillary tube in a manner similar to that previously reported $[9,10]$. Other key aspects of the NV-NMR setup are not shown, including the electromagnet, green laser, optics, and microwave antenna.

(unbound) substrate, and the SABRE active spin-order transfer catalyst. During the lifetime of the catalyst (on the order of milliseconds), spin order can flow from parahydrogen to the substrate (pyridine in Fig. 7). Spin-order transfer is most efficient in an ambient magnetic field of $6.6 \mathrm{mT}$ (details are provided in Appendix B 3). The reversible exchange process and spin-order transfer act together to continually hyperpolarize the free molecular substrate, as long as a steady-state excess of parahydrogen is maintained in the sample solution by periodic bubbling.

\section{Spin-order transfer at level anticrossing}

As a SABRE example, two nuclear spins from the hydrides and five nuclear spins from each of two pyridine
SABRE precatalyst

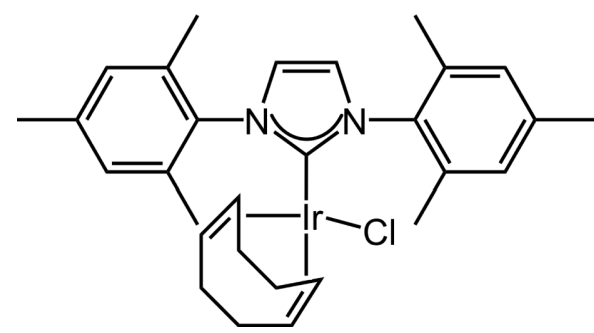

Activation

$\mathrm{H}_{2}$

Substrate (Sub)

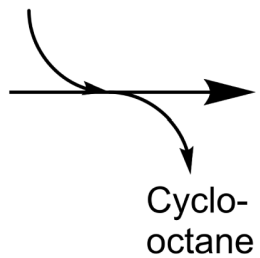

Spin-order transfer catalyst

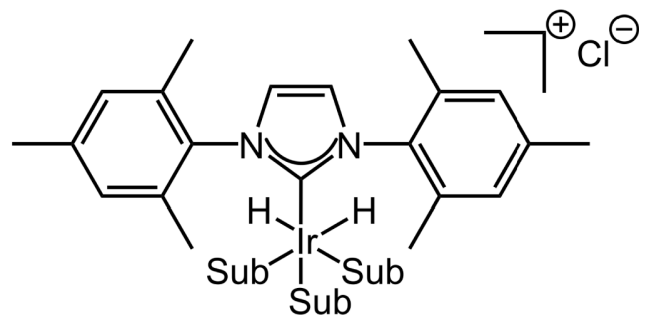

FIG. 6. Chemical activation of the SABRE catalyst precursor to become the SABRE active spin-order transfer catalyst. 


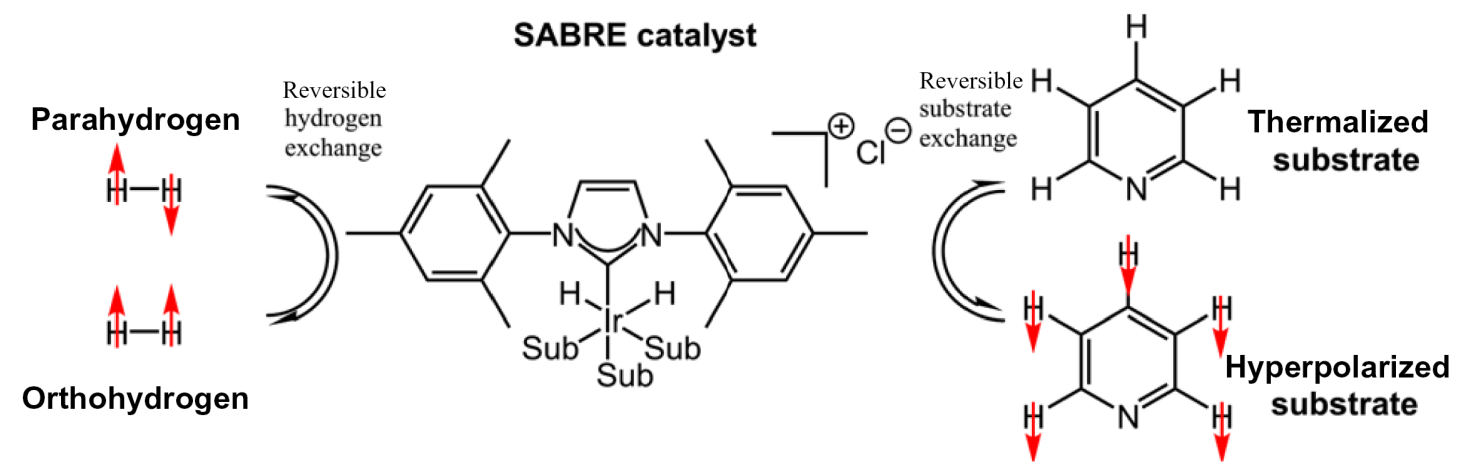

FIG. 7. Reversible hydrogen exchange that leads to hyperpolarization buildup on the free molecular substrate in solution. Left: free (unbound) hydrogen in solution. Center: SABRE active spin-order transfer catalyst. Right: free (unbound) substrate in solution. Steadystate excess of parahydrogen in the sample solution, maintained by periodic bubbling, leads to steady-state excess of hyperpolarized free substrate (Sub).

substrate molecules participate in the spin-order transfer process. Understanding this system of 12 nuclear spins is difficult [17]. A simplified model, with only four spins, nonetheless allows us to analytically predict the resonant magnetic field for the spin-order transfer process with reasonable accuracy (see Fig. 8). To build this model, we begin by constructing the most appropriate eigenstates for the spin system at hand. We choose to combine the singlet $\left(S^{H}\right)$-triplet $\left(T_{+}^{H}, T_{0}^{H}, T_{-}^{H}\right)$ system of the hydride spins with the singlet $\left(S^{S}\right)$-triplet $\left(T_{+}^{S}, T_{0}^{S}, T_{-}^{S}\right)$ system of the substrate spins. As a result, we obtain 16 eigenstates $\left(2^{n}\right.$ with $n=4$ spins), which we name with an uncoupled nomenclature as follows:

$$
\begin{aligned}
& S^{H} S^{S}, S^{H} T_{+}^{S}, S^{H} T_{0}^{S}, S^{H} T_{-}^{S}, \\
& T_{+}^{H} S^{S}, T_{+}^{H} T_{+}^{S}, T_{+}^{H} T_{0}^{S}, T_{+}^{H} T_{-}^{S}, \\
& T_{0}^{H} S^{S}, T_{0}^{H} T_{+}^{S}, T_{0}^{H} T_{0}^{S}, T_{0}^{H} T_{-}^{S}, \\
& T_{-}^{H} S^{S}, T_{-}^{H} T_{+}^{S}, T_{-}^{H} T_{0}^{S}, T_{-}^{H} T_{-}^{S} .
\end{aligned}
$$

The Hamiltonian of the spin system can be expressed in this basis as described in the supplement of Ref. [17].

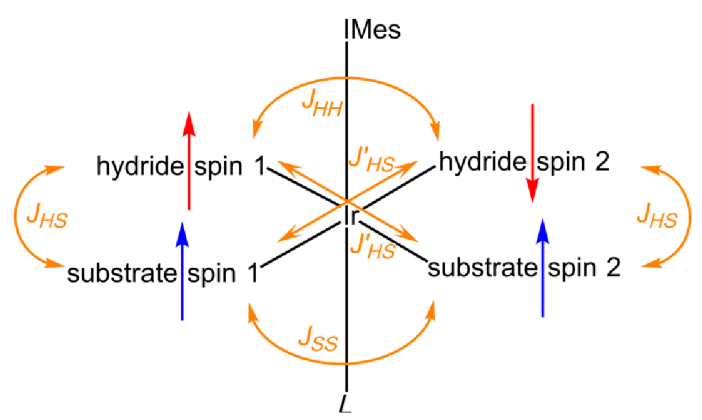

FIG. 8. A simplified spin system that promotes spin-order transfer on the SABRE catalyst. The $J$-coupling values are as follows: $J_{H H} \approx-7 \mathrm{~Hz}, J_{H S}^{\prime} \approx 1.5 \mathrm{~Hz}, J_{H S} \approx 0 \mathrm{~Hz}$, and $J_{H S} \approx 0 \mathrm{~Hz}$.
The eigenstates responsible for the spin-order transfer at level anticrossing are $S^{H} S^{S}$ and $T_{+}^{H} T_{-}^{S}$. These states are chosen because they illustrate a connection between the parahydrogen-derived singlet state on the hydrides $\left(S^{H}\right)$ with a magnetization state on the substrate $\left(T_{-}^{S}\right)$. The states are connected in a $2 \times 2$ block of the Hamiltonian as follows:

$$
\begin{array}{cc}
\left|S^{H} S^{S}\right\rangle \\
\left|T_{+}^{H} T_{-}^{S}\right\rangle
\end{array}\left[\begin{array}{cc}
-S_{H H}+J_{S S} & \frac{\left.\Delta T_{+}^{H} T_{-}^{S}\right\rangle}{2} \\
\frac{\Delta J_{H S}}{2} & v_{H}-v_{S}-\Sigma J_{H S}
\end{array}\right]
$$

where $J_{H H}$ is the $J$ coupling between the hydrides, $J_{S S}$ is the $J$ coupling between the substrate spins, $v_{S}$ is the Larmor frequency of the substrate spins, $v_{H}$ is the Larmor frequency of the hydride spins, $\Delta J_{H S}=J_{H S}-J_{H S}^{\prime}$, and $\Sigma J_{H S}=J_{H S}+J_{H S}^{\prime}$. When the diagonal matrix elements are equal to one another, level anticrossing is established and $\Delta J_{H S}$ can most efficiently transfer spin order from the hydride singlet state $S^{H}$ to the substrate $T_{-}^{S}$ state.

With this Hamiltonian we can now, from first principles, determine the magnetic field at which spin-order flow is most efficient, as follows. Equating the diagonal elements gives

$$
-J_{H H}+J_{S S}=v_{H}-v_{S}-\Sigma J_{H S} .
$$

Solving for the frequency difference $v_{H}-v_{S}$ in Eq. (B1) gives

$$
v_{H}-v_{S}=\Sigma J_{H S}-J_{H H}+J_{S S} .
$$

The frequency difference $v_{H}-v_{S}$ also depends on the magnetic field $B$ as

$$
v_{H}-v_{S}=\gamma B\left(\delta_{H}-\delta_{S}\right)
$$


TABLE I. Experimental protocol.

\begin{tabular}{|c|c|c|c|c|c|}
\hline & Steps & Location & Duration & Temperature & Technical challenges \\
\hline & $\begin{array}{c}\text { Sample } \\
\text { preparation }\end{array}$ & $\ldots$ & $\ldots$ & $\begin{array}{c}\text { Room } \\
\text { temperature }\end{array}$ & $\begin{array}{l}\text { Performed in argon } \\
\text { environment to inhibit oxygen } \\
\text { degradation of catalyst }\end{array}$ \\
\hline \multirow[t]{4}{*}{ Preparation } & $\begin{array}{l}\text { Parahydrogen } \\
\text { generation }\end{array}$ & $\begin{array}{l}\text { Inside coiled copper } \\
\text { tube filled with } \\
{[\mathrm{FeO}(\mathrm{OH})]}\end{array}$ & $\sim 1 \mathrm{~min}$ & $\sim 77 \mathrm{~K}$ & $\ldots$ \\
\hline & $\begin{array}{l}\text { Catalyst } \\
\text { activation }\end{array}$ & $\begin{array}{l}\text { Inside the } \\
\text { experimental } \\
\text { cuvette }\end{array}$ & $20 \mathrm{~min}$ & $\begin{array}{c}\text { Room } \\
\text { temperature }\end{array}$ & \\
\hline & $\begin{array}{l}\text { Parahydrogen } \\
\text { bubbling }\end{array}$ & & $30 \mathrm{~s}$ & & $\begin{array}{l}\text { Gastight seals used to avoid } \\
\text { parahydrogen leak and sample } \\
\text { evaporation }\end{array}$ \\
\hline & Waiting time & & $1 \mathrm{~s}$ & & \\
\hline \multirow[t]{2}{*}{$\begin{array}{l}\text { NMR } \\
\text { measurement }\end{array}$} & $\begin{array}{l}\text { CASR } \\
\text { NV-NMR } \\
\text { detection }\end{array}$ & $\begin{array}{l}\text { Inside the } \\
\text { experimental cuvette }\end{array}$ & $2 \mathrm{~s}$ & $\begin{array}{l}\text { Room } \\
\text { temperature }\end{array}$ & $\begin{array}{l}\text { Optical beam applied in total } \\
\text { internal reflection configuration to } \\
\text { minimize sample heating }\end{array}$ \\
\hline & Waiting time & & $5 \mathrm{~s}$ & & \\
\hline
\end{tabular}

where $\gamma$ is the gyromagnetic ratio of hydrogen, and $\delta_{H}$ and $\delta_{S}$ are the chemical shifts of the hydrides and the substrate, respectively. Solving for the magnetic field $B$ in Eq. (B3) yields

$$
B=\frac{v_{H}-v_{S}}{\gamma\left(\delta_{H}-\delta_{S}\right)} .
$$

Comparing Eqs. (B2) and (B4), we obtain

$$
B=\frac{\Sigma J_{H S}-J_{H H}+J_{S S}}{\gamma(\Delta \delta)}
$$

where $\Delta \delta=\delta_{H}-\delta_{S}$.

For our SABRE system, $\Sigma J_{H S}=1.5 \mathrm{~Hz}, J_{H H}=7 \mathrm{~Hz}$, $J_{S S}=0$, and $\Delta \delta=30 \mathrm{ppm}$. Substituting theses values in Eq. (B5) gives $6.65 \mathrm{mT}$, the ambient magnetic field at which hyperpolarization is most efficient. The experiments reported in the main text are implemented at a magnetic field of $6.576 \mathrm{mT}$.

\section{APPENDIX C: EXPERIMENTAL METHODS}

Details about the NV ensemble sensor, magnetic bias field stabilization, NMR drive coils, synchronized readout protocol, and data analysis are described in the "Methods" section in Ref. [9]. The 200-mW optical beam $(\lambda=$ $532 \mathrm{~nm}$ ), generated by a solid-state laser (Coherent Verdi G7), is focused down to a spot size of about $15 \mu \mathrm{m}$ and pulsed by means of an acousto-optic modulator (IntraAction ASM802B47). The duration of each optical pulse is $5 \mu \mathrm{s}$. The NV spin-state-dependent fluorescence is read out for $1 \mu \mathrm{s}$, followed by optical reinitialization of the NV electronic spins for $4 \mu \mathrm{s}$.

The experimental sequence for the SABRE hyperpolarization with CASR NV-NMR detection is illustrated in Fig. 1(d). Shortly after preparation of the sample, parahydrogen is dispersed into the sample solution for $20 \mathrm{~min}$ to activate the SABRE catalyst. This step is performed only once. Once the catalyst is activated, parahydrogen is bubbled into the sample for $30 \mathrm{~s}$, before every CASR NVNMR measurement, to hyperpolarize the proton nuclear spins in the sample. After a waiting time of $1 \mathrm{~s}$ for the bubbles and any inhomogeneities in the sample or vibrations in the setup to settle, NV-NMR detection of the hyperpolarized sample is performed with use of the CASR measurement protocol [9].

A CASR NV-NMR measurement is summarized here. First, a $\pi / 2 \mathrm{rf}$ pulse (approximately $280 \mathrm{kHz}$ ) with a duration of $100 \mu$ s is applied by the NMR drive coils, resonant with the proton spins of the sample, to induce FNP. The resulting FNP signal from the sample is then measured by CASR NV-NMR detection. The CASR pulse sequence, applied to the NV ensemble spins, is programmed on an arbitrary waveform generator (Tektronix AWG 7122C) and triggered by a pulse generator (Spincore PulseBlaster ESR-PRO $500 \mathrm{MHz}$ ). The CASR pulse sequence consists of interspersed blocks of identical XY8-1 subsequences, each followed by optical readout of the ensemble NV spins, with a total duration of about $25 \mu$ s per XY8-1 subsequence and readout. The $\pi$ and $\pi / 2$ pulse durations used in the XY8-1 subsequence are 60 and $30 \mathrm{ns,}$, respectively. To remove laser and microwave noise, the last 


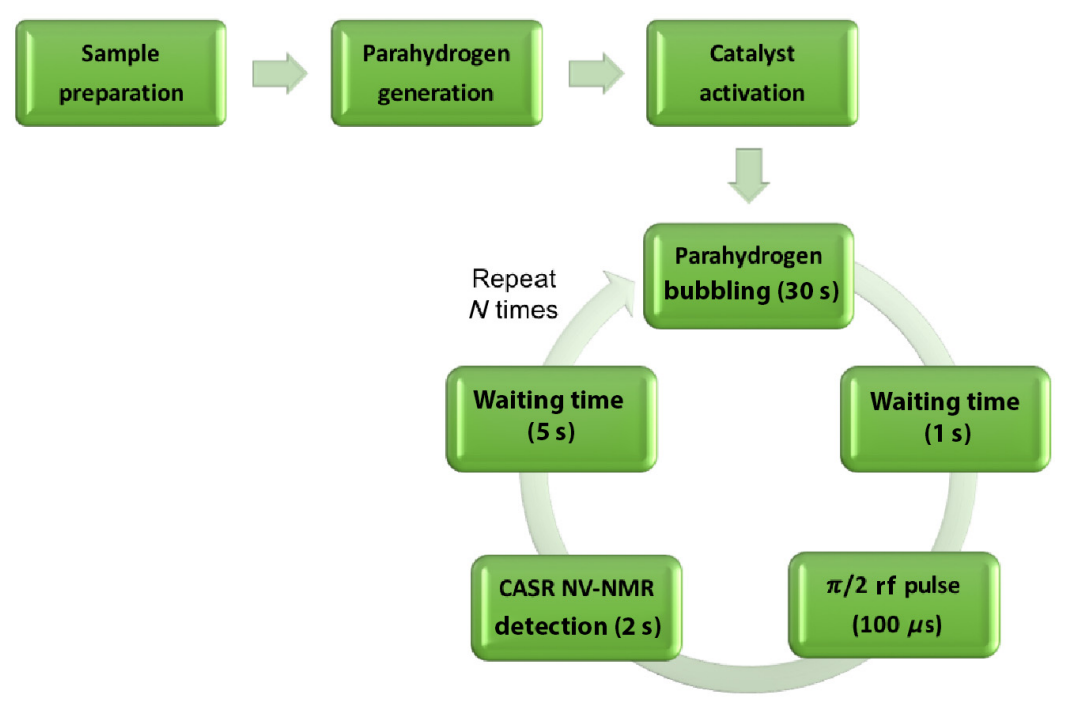

FIG. 9. Experimental protocol. Experimental process such as sample preparation, parahydrogen generation, and catalyst activation are performed only once. NMR measurement, including parahydrogen bubbling and CASR NV-NMR measurement, can be repeated $N$ times, where $N$ is the number of averages used to increase the SNR.

$\pi / 2$ pulse on every alternate XY8- 1 subsequence is phase shifted by $\pi$ and the NV fluorescence measurements from the alternating XY8-1 subsequences are subtracted from each other. Hence two consecutive XY8-1 subsequences (approximately $50 \mu \mathrm{s}$ ) yield one data point of CASR NVNMR detection, with a total measurement typically consisting of 40000 points (total measurement time approximately $2 \mathrm{~s}$ ). After a CASR NV-NMR measurement, there is a waiting time of $5 \mathrm{~s}$ (approximately $T_{1}$ of the hyperpolarized proton spins in the sample) before the start of the next experimental sequence (i.e., $30 \mathrm{~s}$ of parahydrogen bubbling, waiting time of $1 \mathrm{~s}$, and CASR NV-NMR measurement for $2 \mathrm{~s}$ ). The experimental protocol is summarized in Table I and Fig. 9. Table I also lists the key methods used to overcome the technical challenges for each step.

\section{APPENDIX D: DETERMINATION OF SABRE NV-NMR SIGNAL ENHANCEMENT}

Determination of the NMR signal enhancement using SABRE hyperpolarization of the NV ensemble magnetometer is done in two steps: (i) measuring the NV-NMR signal amplitude with the SABRE technique; and (ii) calculating the expected NV-NMR signal amplitude without SABRE.

\section{Measurement of the NV-NMR signal amplitude with SABRE}

We measure the magnitude of the NV-NMR signal amplitude by comparing the CASR fluorescence signal from the SABRE-hyperpolarized sample, as discussed in the main text, with the CASR signal from an ac magnetic field generated from a test coil. The ac magnetic field generated by the test coil is first calibrated, as summarized here and in Ref. [9]. The ac test field is of the form

$$
b(t)=b_{\text {ac }} \sin \left(2 \pi f_{\text {coil }}+\phi\right),
$$

where $b_{\mathrm{ac}}$ is the magnetic field amplitude that needs to be calibrated, $f_{\text {coil }}=280 \mathrm{kHz}$ is the drive frequency, which is within a few kilohertz of the frequency $f_{0}$ used in CASR NV-NMR measurements, and $\phi$ is the phase with respect to the first CASR magnetometry sequence. The field amplitude $b_{\text {ac }}$ is linearly proportional to the voltage $V_{c}$ supplied to the test coil antenna. The CASR fluorescence signal from the ac test field [Fig. 10(a)] is acquired by our varying the test coil drive voltage $V_{c}$ from $0 \mathrm{~V}$ (purple trace) to $0.6 \mathrm{~V}$ (magenta trace), thereby varying the test field amplitude $b_{\text {ac }}$. The CASR fluorescence signal amplitude at $t=$ $0.302 \mathrm{~s}$ in Fig. 10(a) (along the dotted line) is plotted as a function of voltage $V_{c}$ in Fig. 10(b) (blue circles). The sinusoidal fit to the data [blue line in Fig. 10(b)] results in the test signal calibration of 5.7(2) $\mu \mathrm{T} / \mathrm{V}$. For the experiments presented in the main text, we apply a test signal with voltage $V_{c}=1 \mathrm{mV}$, which corresponds to the test signal amplitude of 5.7(2) nT [Fig. 10(c), peak B]. Comparison of the measured CASR signal from pyridine [Fig. 10(c), peak A] with this test signal yields an NMR signal amplitude of 7.1(1) nT.

\section{Calculation of the NV-NMR signal amplitude without SABRE}

The calculated thermal NV-NMR signal amplitude (i.e., without SABRE hyperpolarization) of protons in pure water at $88 \mathrm{mT}$ and room temperature corresponds to $95 \mathrm{pT}$ for our experimental setup [9]. The proton molar concentration of pure water is $111 \mathrm{M}$. Hence for a $100 \mathrm{mM}$ sample concentration, the expected NV-NMR signal amplitude is $85.6 \mathrm{fT}$. Our experiments are performed in a static magnetic field of $6.576 \mathrm{mT}$. Since the thermal spin polarization 
(a)

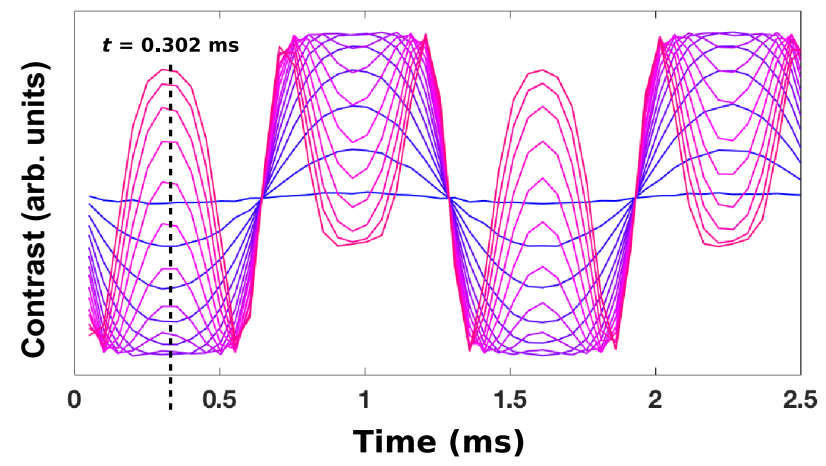

(b)

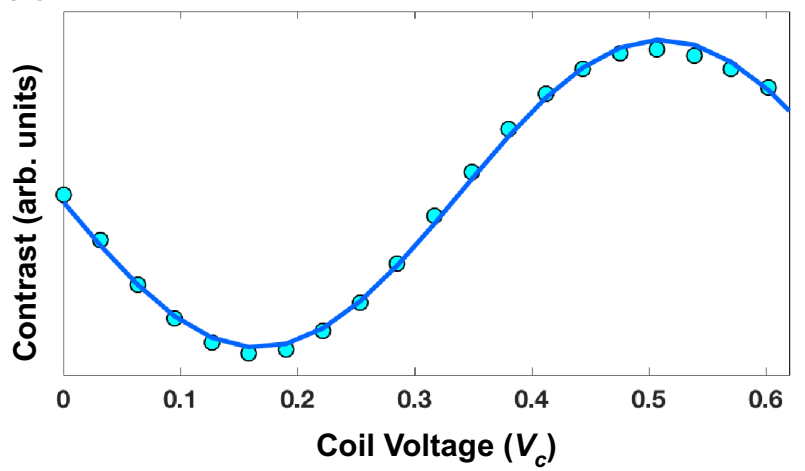

(c)

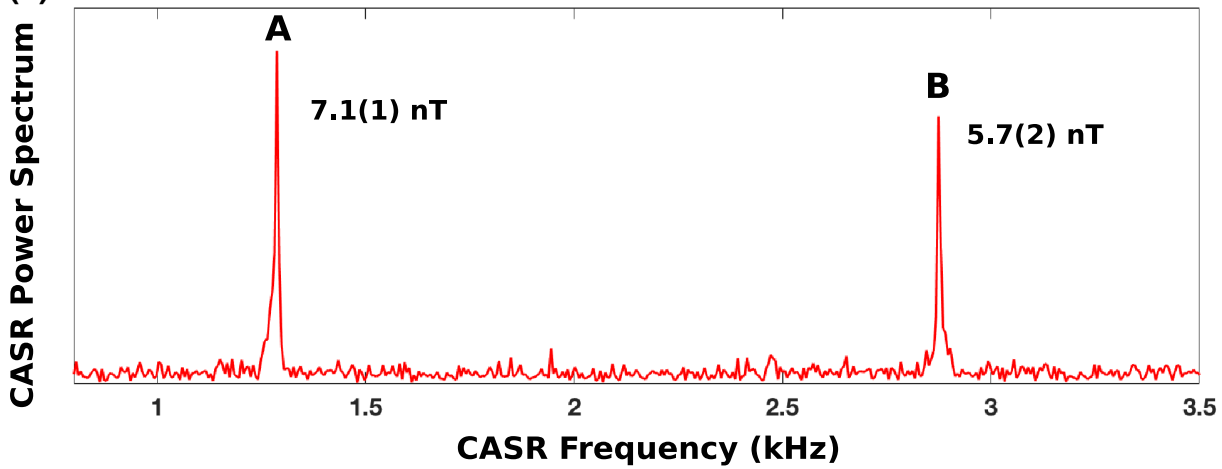

FIG. 10. (a) CASR NV measurements of the ac magnetic test signals from a nearby coil at $f_{\text {coil }}=280 \mathrm{kHz}$. The coil drive voltage $V_{c}$ for the ac signal source of the coil is varied from $V_{c}=0$ (purple trace) to $V_{c}=0.6 \mathrm{~V}$ (magenta trace). (b) CASR signal data (blue points) as a function of coil voltage $V_{c}$ at time $t=0.302 \mathrm{~ms}$, obtained along the dashed line in (a). The blue line is a sinusoidal fit to the data, which gives the magnetic test signal calibration of 5.7(2) $\mu \mathrm{T} / \mathrm{V}$. (c) CASR-detected NV-NMR spectra (peak A) of pyridine and test signal (peak B).

is linearly proportional to the magnetic field in which the experiment is performed, the expected NV-NMR signal amplitude at $6.576 \mathrm{mT}$ corresponds to $6.4 \mathrm{fT}$. Since pyridine has five protons, the expected NV-NMR signal amplitude for thermally polarized pyridine at $100 \mathrm{mM}$ concentration is approximately $32 \mathrm{fT}$.

\section{SABRE NV-NMR signal enhancement}

We determine a $2.22(3) \times 10^{5}$ enhancement in NV-NMR signal amplitude due to SABRE hyperpolarization for a $100 \mathrm{mM}$ pyridine sample by taking the ratio of the measured NV-NMR signal amplitude of $100 \mathrm{mM}$ pyridine with SABRE (from Appendix D 1) to the calculated NV-NMR signal amplitude of $100 \mathrm{mM}$ pyridine without SABRE (from Appendix D 2). This signal amplitude enhancement due to SABRE corresponds to a proton spin polarization of $0.503(7) \%$.

[1] J. M. Taylor, P. Cappellaro, L. Childress, L. Jiang, D. Budker, P. R. Hemmer, A. Yacoby, R. Walsworth, and
M. D. Lukin, High-sensitivity diamond magnetometer with nanoscale resolution, Nat. Phys. 4, 810 (2008).

[2] J. F. Barry, J. M. Schloss, E. Bauch, M. J. Turner, C. A. Hart, L. M. Pham, and R. L. Walsworth, Sensitivity optimization for NV-diamond magnetometry, Rev. Mod. Phys. 92, 015004 (2020).

[3] T. Staudacher, F. Shi, S. Pezzagna, J. Meijer, J. Du, C. A. Meriles, F. Reinhard, and J. Wrachtrup, Nuclear Magnetic Resonance Spectroscopy on a (5-Nanometer)3 Sample Volume, Science 339, 561 (2013).

[4] H. J. Mamin, M. Kim, M. H. Sherwood, C. T. Rettner, K. Ohno, D. D. Awschalom, and D. Rugar, Nanoscale Nuclear Magnetic Resonance with a Nitrogen-Vacancy Spin Sensor, Science 339, 557 (2013).

[5] C. Müller, X. Kong, J.-M. Cai, K. Melentijević, A. Stacey, M. Markham, D. Twitchen, J. Isoya, S. Pezzagna, J. Meijer, J. F. Du, M. B. Plenio, B. Naydenov, L. P. McGuinness, and F. Jelezko, Nuclear magnetic resonance spectroscopy with single spin sensitivity, Nat. Commun. 5, 4703 (2014).

[6] A. O. Sushkov, I. Lovchinsky, N. Chisholm, R. L. Walsworth, H. Park, and M. D. Lukin, Magnetic Resonance Detection of Individual Proton Spins Using Quantum Reporters, Phys. Rev. Lett. 113, 197601 (2014).

[7] I. Lovchinsky, A. O. Sushkov, E. Urbach, N. P. de Leon, S. Choi, K. De Greve, R. Evans, R. Gertner, E. Bersin, C. 
Müller, L. McGuinness, F. Jelezko, R. L. Walsworth, H. Park, and M. D. Lukin, Nuclear magnetic resonance detection and spectroscopy of single proteins using quantum logic, Science 351, 836 (2016).

[8] N. Aslam, M. Pfender, P. Neumann, R. Reuter, A. Zappe, F. Fávaro de Oliveira, A. Denisenko, H. Sumiya, S. Onoda, J. Isoya, and J. Wrachtrup, Nanoscale nuclear magnetic resonance with chemical resolution, Science 357, 67 (2017).

[9] D. R. Glenn, D. B. Bucher, J. Lee, M. D. Lukin, H. Park, and R. L. Walsworth, High-resolution magnetic resonance spectroscopy using a solid-state spin sensor, Nature 555, 351 (2018).

[10] D. B. Bucher, D. R. Glenn, H. Park, M. D. Lukin, and R. L. Walsworth, Hyperpolarization-Enhanced NMR Spectroscopy with Femtomole Sensitivity Using Quantum Defects in Diamond, Phys. Rev. X 10, 021053 (2020).

[11] A. W. Overhauser, Polarization of Nuclei in Metals, Phys. Rev. 92, 411 (1953).

[12] R. W. Adams, J. A. Aguilar, K. D. Atkinson, M. J. Cowley, P. I. P. Elliott, S. B. Duckett, G. G. R. Green, I. G. Khazal, J. López-Serrano, and D. C. Williamson, Reversible Interactions with para-Hydrogen Enhance NMR Sensitivity by Polarization Transfer, Science 323, 1708 (2009).

[13] M. J. Cowley, R. W. Adams, K. D. Atkinson, M. C. R. Cockett, S. B. Duckett, G. G. R. Green, J. A. B. Lohman, R. Kerssebaum, D. Kilgour, and R. E. Mewis, Iridium NHeterocyclic Carbene Complexes as Efficient Catalysts for Magnetization Transfer from para-Hydrogen, J. Am. Chem. Soc 133, 6134 (2011).

[14] P. J. Rayner, M. J. Burns, A. M. Olaru, P. Norcott, M. Fekete, G. G. R. Green, L. A. R. Highton, R. E. Mewis, and S. B. Duckett, Delivering strong $1 \mathrm{H}$ nuclear hyperpolarization levels and long magnetic lifetimes through signal amplification by reversible exchange, Proc. Natl. Acad. Sci. 114, E3188 (2017).

[15] T. Theis, M. P. Ledbetter, G. Kervern, J. W. Blanchard, P. J. Ganssle, M. C. Butler, H. D. Shin, D. Budker, and A. Pines, Zero-Field NMR Enhanced by Parahydrogen in Reversible Exchange, J. Am. Chem. Soc 134, 3987 (2012).

[16] Q. Gong, A. Gordji-Nejad, B. Blümich, and S. Appelt, Trace Analysis by Low-Field NMR: Breaking the Sensitivity Limit, Anal. Chem. 82, 7078 (2010).

[17] T. Theis, M. L. Truong, A. M. Coffey, R. V. Shchepin, K. W. Waddell, F. Shi, B. M. Goodson, W. S. Warren, and E. Y. Chekmenev, Microtesla SABRE Enables 10\% Nitrogen15 Nuclear Spin Polarization, J. Am. Chem. Soc 137, 1404 (2015).

[18] D. B. Bucher, D. P. L. Aude Craik, M. P. Backlund, M. J. Turner, O. Ben Dor, D. R. Glenn, and R. L. Walsworth, Quantum diamond spectrometer for nanoscale NMR and ESR spectroscopy, Nat. Protoc. 14, 2707 (2019).

[19] V. Badilita, R. C. Meier, N. Spengler, U. Wallrabe, M. Utz, and J. G. Korvink, Microscale nuclear magnetic resonance: a tool for soft matter research, Soft Matter 8, 10583 (2012).

[20] J. F. P. Colell, A. W. J. Logan, Z. Zhou, R. V. Shchepin, D. A. Barskiy, G. X. Ortiz, Q. Wang, S. J. Malcolmson, E. Y. Chekmenev, W. S. Warren, and T. Theis, Generalizing, Extending, and Maximizing Nitrogen-15 Hyperpolarization
Induced by Parahydrogen in Reversible Exchange, J. Phys. Chem. C 121, 6626 (2017).

[21] M. L. Truong, T. Theis, A. M. Coffey, R. V. Shchepin, K. W. Waddell, F. Shi, B. M. Goodson, W. S. Warren, and E. Y. Chekmenev, 15N Hyperpolarization by Reversible Exchange Using SABRE-SHEATH, J. Phys. Chem. C 119, 8786 (2015).

[22] S. Lehmkuhl, M. Suefke, A. Kentner, Y.-F. Yen, B. Blümich, M. S. Rosen, S. Appelt, and T. Theis, SABRE polarized low field rare-spin spectroscopy, J. Chem. Phys. 152, 184202 (2020).

[23] P. Ellinger and M. M. Kader, Nicotinamide metabolism in mammals, Biochem. J. 44, 77 (1949).

[24] C. Cantó, R. H. Houtkooper, E. Pirinen, D. Y. Youn, M. H. Oosterveer, Y. Cen, P. J. Fernandez-Marcos, H. Yamamoto, P. A. Andreux, P. Cettour-Rose, K. Gademann, C. Rinsch, K. Schoonjans, A. A. Sauve, and J. Auwerx, The $\mathrm{NAD}(+)$ precursor nicotinamide riboside enhances oxidative metabolism and protects against high-fat diet-induced obesity, Cell Metab. 15, 838 (2012).

[25] A. A. Sauve, NAD+ and Vitamin B3: From Metabolism to Therapies, J. Pharmacol. Exp. Ther. 324, 883 (2008).

[26] S. Knecht, A. S. Kiryutin, A. V. Yurkovskaya, and K. L. Ivanov, Re-polarization of nuclear spins using selective SABRE-INEP, J. Magn. Reson. 287, 10 (2018).

[27] K. D. Atkinson, M. J. Cowley, S. B. Duckett, P. I. P. Elliott, G. G. R. Green, J. López-Serrano, I. G. Khazal, and A. C. Whitwood, Para-Hydrogen Induced Polarization without Incorporation of Para-Hydrogen into the Analyte, Inorg. Chem. 48, 663 (2009).

[28] T. Theis, M. Truong, A. M. Coffey, E. Y. Chekmenev, and W. S. Warren, LIGHT-SABRE enables efficient inmagnet catalytic hyperpolarization, J. Magn. Reson. 248, 23 (2014).

[29] S. S. Roy, G. Stevanato, P. J. Rayner, and S. B. Duckett, Direct enhancement of nitrogen-15 targets at highfield by fast ADAPT-SABRE, J. Magn. Reson. 285, 55 (2017).

[30] A. Svyatova, I. V. Skovpin, N. V. Chukanov, K. V. Kovtunov, E. Y. Chekmenev, A. N. Pravdivtsev, J.-B. Hövener, and I. V. Koptyug, 15N MRI of SLIC-SABRE Hyperpolarized 15N-Labelled Pyridine and Nicotinamide, Chem.-A Eur. J. 25, 8465 (2019).

[31] A. N. Pravdivtsev, A. V. Yurkovskaya, H.-M. Vieth, and K. L. Ivanov, RF-SABRE: A Way to Continuous Spin Hyperpolarization at High Magnetic Fields, J. Phys. Chem. B 119, 13619 (2015).

[32] T. Theis, N. M. Ariyasingha, R. V. Shchepin, J. R. Lindale, W. S. Warren, and E. Y. Chekmenev, Quasi-Resonance Signal Amplification by Reversible Exchange, J. Phys. Chem. Lett. 9, 6136 (2018).

[33] N. M. Ariyasingha, J. R. Lindale, S. L. Eriksson, G. P. Clark, T. Theis, R. V. Shchepin, N. V. Chukanov, K. V. Kovtunov, I. V. Koptyug, W. S. Warren, and E. Y. Chekmenev, Quasi-Resonance Fluorine-19 Signal Amplification by Reversible Exchange, J. Phys. Chem. Lett. 10, 4229 (2019).

[34] L. Bordonali, N. Nordin, E. Fuhrer, N. MacKinnon, and J. G. Korvink, Parahydrogen based NMR hyperpolarisation 
goes micro: an alveolus for small molecule chemosensing, Lab Chip 19, 503 (2019).

[35] J. Smits, J. T. Damron, P. Kehayias, A. F. McDowell, N. Mosavian, I. Fescenko, N. Ristoff, A. Laraoui, A. Jarmola, and V. M. Acosta, Two-dimensional nuclear magnetic resonance spectroscopy with a microfluidic diamond quantum sensor, Sci. Adv. 5 (2019).
[36] P. J. Rayner and S. B. Duckett, Signal Amplification by Reversible Exchange (SABRE): From Discovery to Diagnosis, Angew. Chem. 57, 6742 (2018).

[37] W. Iali, P. J. Rayner, and S. B. Duckett, Using parahydrogen to hyperpolarize amines, amides, carboxylic acids, alcohols, phosphates, and carbonates, Sci. Adv. 4 (2018). 